\title{
Growth and Self-Ejection of Single Condensate Droplet on Nanostructured Microcones
}

\author{
Nicolò G. Di Novo ${ }^{1,2}$, Alvise Bagolini ${ }^{2}$, Nicola M. Pugno ${ }^{1,3}$ \\ ${ }^{1}$ Laboratory of Bio-Inspired, Bionic, Nano, Meta, Materials \& Mechanics, Department of Civil, Environmental and \\ Mechanical Engineering, University of Trento, Via Mesiano, 77, 38123 Trento, Italy \\ ${ }^{2}$ Sensors and Devices Center, FBK, Via Sommarive 18, 38123 Trento \\ ${ }^{3}$ School of Engineering and Materials Science, Queen Mary University of London, Mile End Road, London E1 4NS, \\ United Kingdom \\ nicolo.dinovo@unitn.it; bagolini@ fbk.eu; nicola.pugno@unitn.it
}

\section{Extended Abstract}

In the last decade, the phenomenon of coalescence-induced droplet jumping has been studied in depth because of the advantages it brings in applications such as anti-frosting, anti-icing, condensation heat transfer, water harvesting, thermal diodes for electronics cooling and self-cleaning [1]. In this work we theoretically and experimentally show that surfaces structured with micro truncated cones covered with highly hydrophobic nanostructures exhibit a recurrent self-ejection of single condensate droplets in addition to the common coalescence-induced jumps. We analytically modelled the external forces acting both on a droplet of condensate slowly growing in conical pores (a simplification of the growth between truncated cones) and during the following rapid transient for two possible cases of self-ejection: 1) rapid swelling out of the structures and 2) detachment from a pinning site (a less hydrophobic defect or the nucleation site). Viscous and adhesion dissipations were included in the modelling. We described the shape of the growing droplet as a function of the tapering $(\beta)$ and of dynamic contact angles $\left(\vartheta_{a d v}\right.$ and $\left.\vartheta_{r e c}\right)$. For both cases, the minimum requirement for self-ejection is that the receding angle of the walls $\left(\vartheta_{\text {rec }}\right)$ and any pinning site $\left(\vartheta_{\text {rec }}^{\text {pinning }}\right)$ are greater than $90^{\circ}+\beta$. In the first case, as the volume increases, the upper meniscus moves towards the apex of the cones and the lower one follows it as soon as it reaches $\vartheta_{\text {rec }}$; when the droplet reaches the apex of the structures it self-ejects because the superior meniscus expands rapidly and a Laplace pressure gradient is generated between the menisci. In the second case, the superior meniscus grows towards the apex and when the lower one recedes from the pinning site, the droplet self-ejects due to the Laplace pressure gradient generated by the abrupt change in wettability, as recently reported for relatively large droplets (radius $>50 \mu \mathrm{m}$ ) in diverging grooves [2]. Describing growth and self-ejection with a system of forces requires a careful distinction between external and internal forces. The modelling with forces, unlike the energetic one [2], allowed us to describe the ejection transient dynamics and to derive the ejection velocity while maintaining the dependence on the dynamic contact angles of the superhydrophobic walls and of an eventual pinning site, fundamental quantities for the design of real surfaces. The analytical results relative to the case of the pinning site foresee that, at a fixed contact angle hysteresis of the superhydrophobic walls and $\vartheta_{\text {rec }}^{\text {pinning }}$, the ejection velocity increases with $\beta$ (until the limit $\beta=\vartheta_{\text {rec }}^{\text {pinning }}-90^{\circ}$, beyond which there cannot be ejection); in addition, the velocity is greater as the droplet size decreases if viscous dissipations are not considered while a peak trend is observed with the dissipations included.

We fabricated silicon truncated micro cones arranged in square and hexagonal patterns and covered with nanostructures. Through condensation experiments in a controlled environmental chamber we observed for the first time the self-ejection of the drops that nucleate and grow between the cones (in the case in question the droplet diameter $\approx 11 \mu \mathrm{m}$ ). By fabricating cones of different sizes, we have also studied the self-ejection rate per unit area as a function of the droplet size. Furthermore, we performed preliminary condensation frosting experiments. Future investigations of this new class of jumping droplet surfaces may show important advantages in the mentioned applications.

\section{References}

[1] Chuntian Liu et al., Langmuir 202137 (3), 983-1000, doi: 10.1021/acs.langmuir.0c02758

[2] Xiao Yan et al., "Laplace Pressure Driven Single-Droplet Jumping on Structured Surfaces,"ACS Nano 202014 (10), 12796-12809, doi: 10.1021/acsnano.0c03487 